Article

\title{
Revisiting Chiral Recognition Mechanism on Chicken Alpha 1-Acid Glycoprotein: Location of Chiral Binding Sites and Insight into Chiral Binding Mechanism
}

\author{
Jun Haginaka ${ }^{1, *(\mathbb{D}}$, Taku Yamashita ${ }^{2}$, Hirofumi Tsujino ${ }^{3}$ and Mitsuhiro Arisawa ${ }^{3}$ \\ 1 Institute for Biosciences, Mukogawa Women's University, 11-68, Koshien Kyuban-cho, \\ Nishinomiya 663-8179, Japan \\ 2 School of Pharmacy and Pharmaceutical Sciences, Mukogawa Women's University, 11-68, \\ Koshien Kyuban-cho, Nishinomiya 663-8179, Japan; taku@mukogawa-u.ac.jp \\ 3 Graduate School of Pharmaceutical Sciences, Osaka University, 1-6 Yamadaoka, Suita, Osaka 565-0871, Japan; \\ htsujino@phs.osaka-u.ac.jp (H.T.); arisaw@phs.osaka-u.ac.jp (M.A.) \\ * Correspondence: haginaka@mukogawa-u.ac.jp
}

Citation: Haginaka, J.; Yamashita, T.; Tsujino, H.; Arisawa, M. Revisiting Chiral Recognition Mechanism on Chicken Alpha 1-Acid Glycoprotein: Location of Chiral Binding Sites and Insight into Chiral Binding Mechanism. Separations 2021, 8, 73. https:// doi.org/10.3390/separations8060073

Academic Editor: Victoria Samanidou

Received: 6 April 2021

Accepted: 19 May 2021

Published: 23 May 2021

Publisher's Note: MDPI stays neutral with regard to jurisdictional claims in published maps and institutional affiliations.

Copyright: (c) 2021 by the authors. Licensee MDPI, Basel, Switzerland. This article is an open access article distributed under the terms and conditions of the Creative Commons Attribution (CC BY) license (https:// creativecommons.org/licenses/by/ $4.0 /)$.

\begin{abstract}
Chiral stationary phases based on chicken alpha 1-acid glycoprotein (cAGP) have been used for enantioseparations of various compounds. However, the chiral binding sites and mechanism have not been clarified yet. Based on chromatographic properties of native and W26-modified cAGP columns and docking simulations of studied compounds into the generated model structure of cAGP, the chiral binding sites were located on CAGP and the chiral binding mechanism was discussed. On cAGP, there existed a binding cavity lined with H25, W26, Y47, R128, T129, D161 and E168, which contribute electrostatic or hydrogen bonding interactions. Benzoin and chlorpheniramine enantiomers interacted with cAGP at almost the same sites a little away from W26, while propranolol enantiomers docked, slightly shifting toward H25 and W26. Furthermore, in addition to hydrophobic interactions, ionic interactions between amino groups of chlorpheniramine enantiomers and a carboxyl group of D161 or E168 played an important role in the chiral recognition, while hydrophobic interactions and hydrogen bonding interactions worked for the chiral recognition of benzoin and propranolol enantiomers.
\end{abstract}

Keywords: protein-based chiral stationary phase; alpha 1-acid glycoprotein; chiral recognition mechanism; molecular docking

\section{Introduction}

Chiral stationary phases (CSPs) based on proteins have been used for enantioseparations of racemates or chiral compounds [1,2]. Those include serum proteins such as bovine serum albumin [3], human serum albumin [4], human alpha 1-acid glycoprotein (hAGP) [5] and chicken AGP (cAGP) [6,7]; enzymes such as trypsin [8], cellobiohydrolase [9], pepsin [10] and amyloglucosidase [11]; and other proteins such as antibody [12] and fatty acid binding protein [13]. The disadvantages of protein-based CSPs include low capacity, lack of column ruggedness and limited understanding of the chiral recognition mechanism. The advantages are that various drug enantiomers could be separated in reversed-phase mode because of multiple binding interactions such as hydrophobic, hydrogen bonding and electrostatic interactions or multiple binding sites [1]. Among those CSPs, a CSP based on hAGP could separate a wide range of basic, neutral and acidic enantiomers [1]. hAGP consisted of 183 amino acid residues, which contain five $N$-linked glycans, and accounted for the total 37-54 kDa mass [14,15]. It belongs to a member of the lipocalin family, which is a transporter for small hydrophobic molecules $[16,17]$.

Furthermore, hAGP also binds to a variety of drugs, mainly hydrophobic or basic drugs because of its isoelectric point (pI) 2.7-3.8 [16]. Besides the high heterogeneity of glycans, the protein part has also been found to show polymorphism [18]. The variants 
are encoded by two different genes: The F1*S variant is encoded by the alleles of the same gene (orosomucoid1 (ORM1)), while the A variant is encoded by a different gene (ORM2). The molar ratio of the F1*S and A variant hAGPs in blood typically ranges from 3:1 to 2:1 $[19,20]$. The first $X$-ray structural analysis of the F1*S variant hAGP was reported, and its ligand-binding sites, which consist of the lobes I, II and III, were clarified. Lobe I is a large and deep cavity, and provides sufficient spaces for ligand-binding pockets. Lobes II and III are on each side of lobe I and are negatively charged. Furthermore, the x-ray crystal structure of the A variant hAGP, which had only two lobes, lobes I and II, and had narrower binding sites than the F1*S variant hAGP, were determined [21]. The drug bindings to hAGP have been extensively investigated using co-crystals with ligands [22], docking study with the 3D molecular model [23-30], circular dichroism (CD) and fluorescence spectroscopy [23,31,32], photoaffinity labelling [33] and NMR spectroscopy [34]. Although most of studies indicated that the ligand-binding sites were located on lobes I-III, only one study by induced CD spectra suggested that W25 of hAGP was essentially involved in the ligand-bindings [32].

Regarding chiral binding mechanism of ligands on CAGP, a few studies were reported [24,34]. Chiral bindings of the coumarins (warfarin, acenocoumarol and phenprocoumon) to hAGPs of the F1*S and A variants (ORM1 and ORM2, respectively) were examined [24]. The enantiomers of the coumarins were docked onto the F1*S and A variant hAGPs in essentially the same position, including W122, and orientation [24]. (S)Enantiomers of warfarin and acenocoumarol were found to possess a higher affinity for ORM 1 than the corresponding $(R)$-enantiomers in binding measurements by stereoselective analysis of the ultrafiltrates [24]. (R)- and (S)-acenocoumarol to hAGP models of the F1*S and A variants show a slight preference for $(S)$-enantiomer in the case of both variant hAGPs [24]. However, the proposed model could not explain chiral bindings of coumarins onto hAGP. Chiral bindings of propranolol enantiomers to cAGP were investigated with ligand-detected NMR experiments [34]. It was found that each enantiomer bound to the AGP binding pocket in a different orientation.

CSPs based on crude ovomucoid, ovomucoid from chicken (OMCHI), which was isolated from egg whites, were developed [35] and used for enantioseparations of neutral, acidic and basic compounds [36]. However, crude ovomucoid included OMCHI and ovoglycoprotein (OGCHI), whose content was about $10 \%$. Since OMCHI and OGCHI had the same pI 4.1 [1], OMCHI was contaminated with OGCHI in crude ovomucoid preparation [6]. Furthermore, OMCHI had no chiral recognition ability, but the chiral recognition ability of crude ovomucoid came from OGCHI [6]. The amino acid sequence of OGCHI was clarified [7]. OGCHI consisted of 183 amino acids and accounted for the total 30 kDa mass. Furthermore, OGCHI showed 31-32\% identities to rabbit AGP and hAGP [7]. OGCHI was a member of the AGP family identified in mammals, and was chicken AGP (cAGP) [7]. As hAGP, cAGP conserved the number and locations of the S-S bridges (C6-146 and C73-C163), and had five N-linked glycans: N16, 70, 77 and 87 were fully glycosylated and N62 was partially glycosylated [37].

cAGP had only one tryptophan residue at 26 position [37]. W26-modified cAGP completely lost chiral recognition ability for $\beta$-blockers such as propranolol, alprenolol and oxprenolol, while it still conserved lower chiral recognition ability for chlorpheniramine and benzoin, compared with native cAGP [38]. Therefore, it was concluded that $\beta$-blockers bound on a single binding site near the W26 region and that further bindings of chlorpheniramine and benzoin occurred at the secondary binding site [38]. Furthermore, by CD and ultraviolet/visible absorption spectroscopy experiments it was supported that W26 was responsible for the ligand-bindings to cAGP [39].

In this study, we located the chiral binding sites on cAGP and discussed the chiral recognition mechanism of benzoin, chlorpheniramine and propranolol, based on chromatographic properties of native and W26-modified cAGP columns and molecular docking results. 


\section{Materials and Methods}

\subsection{Reagents}

2-Nitrophenylsulfenyl chloride was purchased from Tokyo Chemical Industry (Tokyo, Japan). Other reagents and solvents were obtained from Nacalai Tesque (Kyoto, Japan). All reagents were of an analytical-reagent grade and were used without further purification. The structures of studied compounds were illustrated in Figure 1.<smiles>O=C(c1ccccc1)C(O)c1ccccc1</smiles>

Benzoin<smiles>CN(C)CCC(c1ccc(Cl)cc1)c1ccccn1</smiles>

Chlorpheniramine<smiles>CC(C)NCC(O)COc1cccc2ccccc12</smiles>

Propranolol

Figure 1. Structures of compounds used in this study.

\subsection{Preparation of W26-Modified cAGP}

cAGP was isolated as reported previously [6]. W26-modified cAGP with 2-nitrophenylsulfenyl chloride was obtained according to a previously reported method [40]. Briefly, $3 \mathrm{mg}$ of 2-nitrophenylsulfenyl chloride dissolved in $50 \mu \mathrm{L}$ of glacial acetic acid were added to a $3 \mathrm{~mL}$ of a $20 \mathrm{vol} \%$ acetic acid solution including $100 \mathrm{mg}$ of cAGP. Then the reaction mixture was stirred for $5 \mathrm{~h}$ at $0{ }^{\circ} \mathrm{C}$. The reaction mixture was dialyzed against water for $60 \mathrm{~h}$ at $4{ }^{\circ} \mathrm{C}$ and lyophilized.

\subsection{Preparation of Native and W26-Modified cAGP Columns}

Native and W26-modified cAGP columns were prepared as reported previously [6]. Briefly, $2.66 \mu \mathrm{mol}$ of native and W26-modified cAGPs, respectively, were reacted with $1 \mathrm{~g}$ of aminopropyl-silica gels ( $5 \mu \mathrm{m}$ particle size, $12 \mathrm{~nm}$ pore size) activated with $N, N$ disuccinimidyl carbonate (DSC). Each protein reacted was completely immobilized to DSC-activated aminopropyl-silica gels [41]. The obtained materials were packed into a $2.0 \mathrm{~mm}$ I.D. $\times 100 \mathrm{~mm}$ stainless-steel column by a slurry packing method to evaluate chiral recognition abilities. The flow-rate was maintained at $0.2 \mathrm{~mL} / \mathrm{min}$. Detection was carried out at $210 \mathrm{~nm}$. The retention factor, $k$, and enantioseparation factor, $\alpha$, of a racemate were calculated according to a method reported previously [6]. All separations were performed at $25^{\circ} \mathrm{C}$ using a water bath.

\subsection{Generation of Model Structure of cAGP and Docking Simulations}

The homology model of cAGP was constructed using Swiss-Model server [42]. The crystal structure of hAGP (A variant (ORM2); pdbid, 3apu), which showed the highest score of $28.8 \%$ homology with cAGP in amino acids sequence alignments, was selected, and the model structure was built in accordance with the alignment with the target protein using ProMod3 [43]. In addition, the model structure of cAGP was generated using i-TASSER server [44], and the built structure seemed to be indistinguishable with that from Swiss-Model server. The validated model structure from Swiss-Model server was used for docking simulations as previously reported [45-47]. First, the model structure was prepared by Protein Preparation Wizard, which provides a state for calculation with charge, orientation of groups, etc. Next, the chemical structures for benzoin, chlorpheniramine and propranolol were obtained from Pubchem database (https:/ / pubchem.ncbi.nlm.nih.gov/, accessed on 22 May 2021) for both (R)- and (S)-configurations, and feasible conformations were prepared by LigPrep module. Each prepared ligand was docked to the built model 
structure of cAGP using Glide module using standard precision (SP) mode [48], and the docked structures with the highest glide score for each ligand were visualized with measuring distances among atoms by the PyMOL Molecular Graphics System from Schrödinger (New York, NY, USA). All tools for docking and visualization were from Schrödinger.

\section{Results}

\subsection{Enantioseparations on Native and W26-Modified cAGP Columns}

Since cAGP had only one tryptophan residue at 26 position, W26 of cAGP was only modified with 2-nitrophenylsulfenyl chloride. Table 1 shows retention and enantioseparation factors of benzoin, chlorpheniramine and propranolol on native and W26-modified cAGP columns. Three racemic compounds were enantioseparated on a native cAGP column. Though on a W26-modified cAGP column, retention and enantioseparation factors of benzoin and chlorpheniramine were decreased, they were still enantioseparated. However, propranolol was not enantioseparated on a W26-modified cAGP column along with a drastic decrease in the retention factor. Similarly, chiral resolution of $\beta$-blockers such as alprenolol and pindolol was completely lost on a W26-modified cAGP column [38]. Furthermore, competition studies using $N, N$-dimethyl-n-octylamine (DMOA) as a competitor indicated that propranolol competed with DMOA on a single binding site near the W26 region and that further bindings of benzoin and chlorpheniramine occurred at the secondary binding site in a non-competitive fashion with DMOA [38]. Therefore, it was considered that there existed at least two chiral binding sites on native cAGP.

Table 1. Comparison of chiral recognition abilities of native and W26-modified cAGP columns ${ }^{1}$.

\begin{tabular}{ccccccc}
\hline \multirow{2}{*}{ Entry } & \multicolumn{3}{c}{ Native cAGP Column } & \multicolumn{2}{c}{ W26-modified cAGP Column } \\
\cline { 2 - 6 } & $\boldsymbol{k}_{\mathrm{S}}{ }^{2}$ & $\boldsymbol{k}_{\mathbf{R}}{ }^{2}$ & $\boldsymbol{\alpha}^{3}$ & $\boldsymbol{k}_{\mathrm{S}}{ }^{2}$ & $\boldsymbol{k}_{\mathbf{R}}{ }^{2}$ & $\boldsymbol{\alpha}^{3}$ \\
\hline Benzoin & 5.97 & 19.5 & 3.27 & 4.82 & 8.00 & 1.66 \\
\hline Chlorpheniramine & 8.40 & 4.49 & 1.87 & 4.03 & 2.76 & 1.46 \\
\hline Propranolol & 27.0 & 30.5 & 1.13 & 2.06 & 2.06 & 1.00 \\
\hline
\end{tabular}

${ }^{1}$ Data adapted from Table 1 in [38]. ${ }^{2}$ Retention factors of $(S)$ - and (R)-enantiomers, $k_{S}$ and $k_{R}$, respectively.

${ }^{3}$ Enantioseparation factor of $(S)$ - and $(R)$-enantiomers.

\subsection{Docking Simulations of Studied Compounds into Generated Model Structure of cAGP}

As described above, the model structure of cAGP was generated using hAGP as a template, and then the generated structure was validated with another modeling server. Subsequently, each enantiomer of three compounds, benzoin, chlorpheniramine and propranolol, was simulated and docked to a certain cavity on the generated model structure of cAGP (Figure 2).

(A)

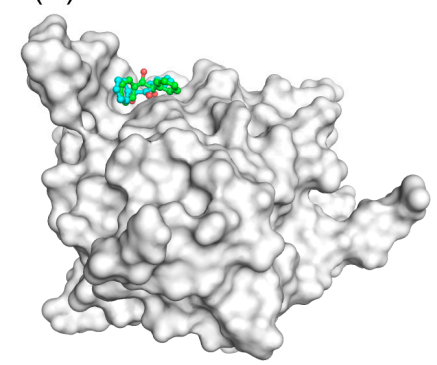

(B)

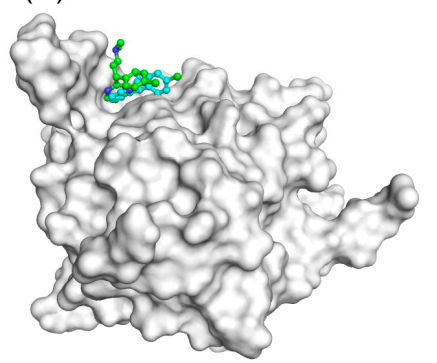

(C)

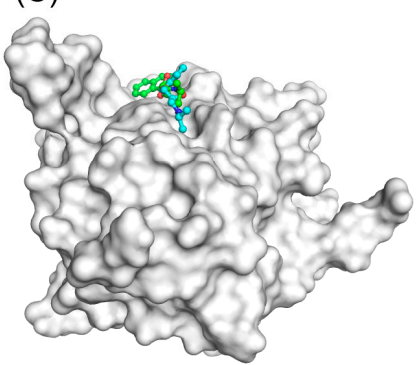

Figure 2. Docking structures of cAGP with (A) benzoin, (B) chlorpheniramine and (C) propranolol enantiomers. The built model structure of cAGP was illustrated as surface mode and the docked enantiomers were shown as ball-and-stick models, respectively. 
Remarkably, each enantiomer of two compounds, benzoin and chlorpheniramine, was located onto a similar position of the surface; however the docked propranolol enantiomers slightly shifted to the relatively small cliff. The differences between $(R)-$ and $(S)$-configurations in the binding site were subsequently investigated in detail.

As shown in Figure 3, both $(R)$ - and $(S)$-benzoin were docked onto a similar position of a cavity of the generated model structure of cAGP. The positions seemed to be fastened by one of aromatic rings in both configurations by hydrophobic interactions. Furthermore, some of hydrogen bonding interactions work for chiral recognition of $(R)$ - and (S)-benzoin. A hydroxyl group of $(R)$-benzoin is close to oxygen atoms in either a carbonyl or hydroxyl group of D161 with a distance of 3.2 or $4.9 \AA$, and a hydroxyl group of Y47 is nearby a carbonyl group of $(R)$-benzoin with a distance of $2.8 \AA$ (Figure $3 \mathrm{~A})$. An oxygen atom in either a carbonyl or hydroxyl group of D161 is also close to a hydroxyl group of $(S)$-benzoin at a distance of $2.8 \AA$; however, Y47 is a bit far from the bound (S)-benzoin at a distance of $5.3 \AA$ (Figure $3 \mathrm{~B}$ ). These results indicate that $(R)$-benzoin binds to cAGP more tightly than (S)-benzoin.

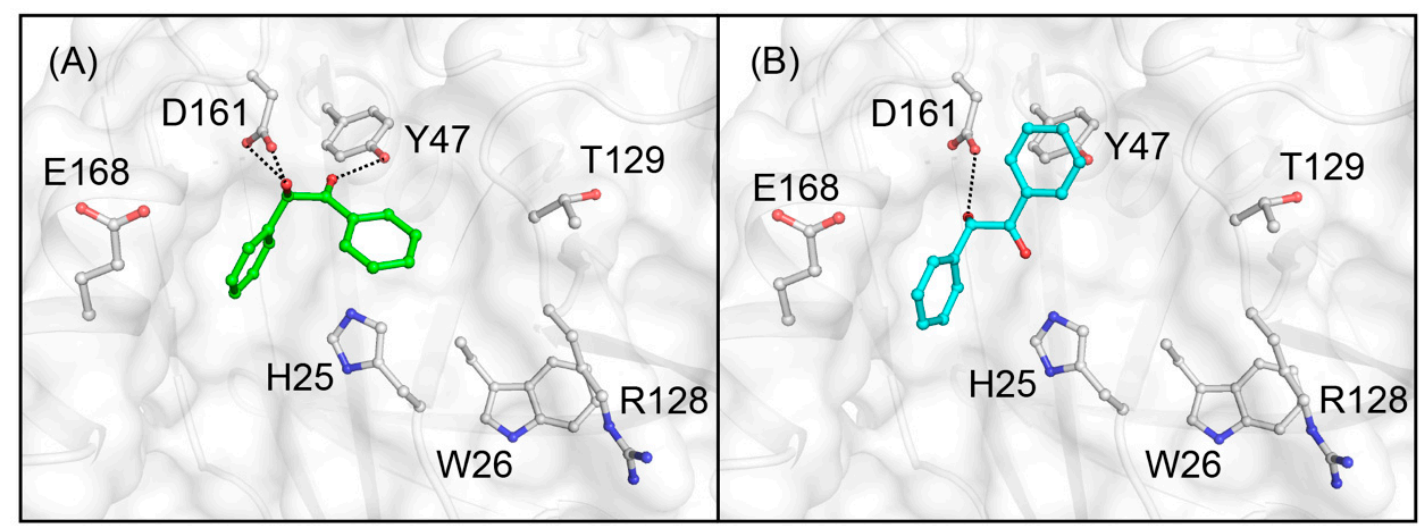

Figure 3. Simulated docking structures of cAGP focusing on the surrounding of bound (A) (R)-benzoin and (B) (S)-benzoin. The docked compounds were represented in either green or cyan for carbon atoms. Besides, nitrogen and oxygen atoms were colored in blue and red, respectively. The remarked amino acid residues were labeled and shown in gray for carbon atoms, and feasible interactions were depicted as dotted lines.

Next, $(R)$ - and (S)-chlorpheniramine were also suggested to bind the same cavity with benzoin (Figure 4). As shown in Figure 4, a carboxyl group of E168 is close to an amino group of $(R)$-chlorpheniramine at a distance of $2.6 \AA$, while an amino group of (S)-chlorpheniramine could interact with a carboxyl group of D161 with $3.0 \AA$ instead of E168 for $(R)$-chlorpheniramine. Electrostatic interactions between amino groups of $(R)$ - and (S)-chlorpheniramine and carboxyl groups of E168 and D161, respectively, could work for the recognition. In addition, as shown in Figure 4B, Y47 was located nearby bound (S)-chlorpheniramine with a distance of $3.9 \AA$ A Furthermore, the chlorine atom of (S)-chlorpheniramine was close to a carbonyl group for main chain between R128 and T129 with a distance of $3.6 \AA$. It implied that $(S)$-chlorpheniramine could interact with the main chain via halogen bonding interactions. These results indicate that $(S)$-chlorpheniramine interacts with cAGP more tightly than $(R)$-chlorpheniramine.

Finally, $(R)$ - and $(S)$-propranolol were again docked to the generated model structure of cAGP (Figure 5). Interestingly, the docked propranolol was slightly shifted toward H25 and W26 with a consequent distance of $4.3 \AA$ to H25. Although Y47 was again suggested to be involved in the binding of $(R)$-propranolol with a distance of $4.7 \AA$, it could be weak to anchor the docked propranolol. A carbonyl group for main chain between R128 and T129 was remarkably located to either 2.7 or $2.8 \AA$ for $(R)$-propranolol or (S)-propranolol. The former hydrogen bonding interactions were with an amino group of $(R)$-propranolol, while the latter hydrogen bonding interactions were with a hydroxyl group of $(S)$-propranolol. 
These results indicate that the $(R)$-propranolol binding to cAGP is more favorable than the (S)-propranolol binding.

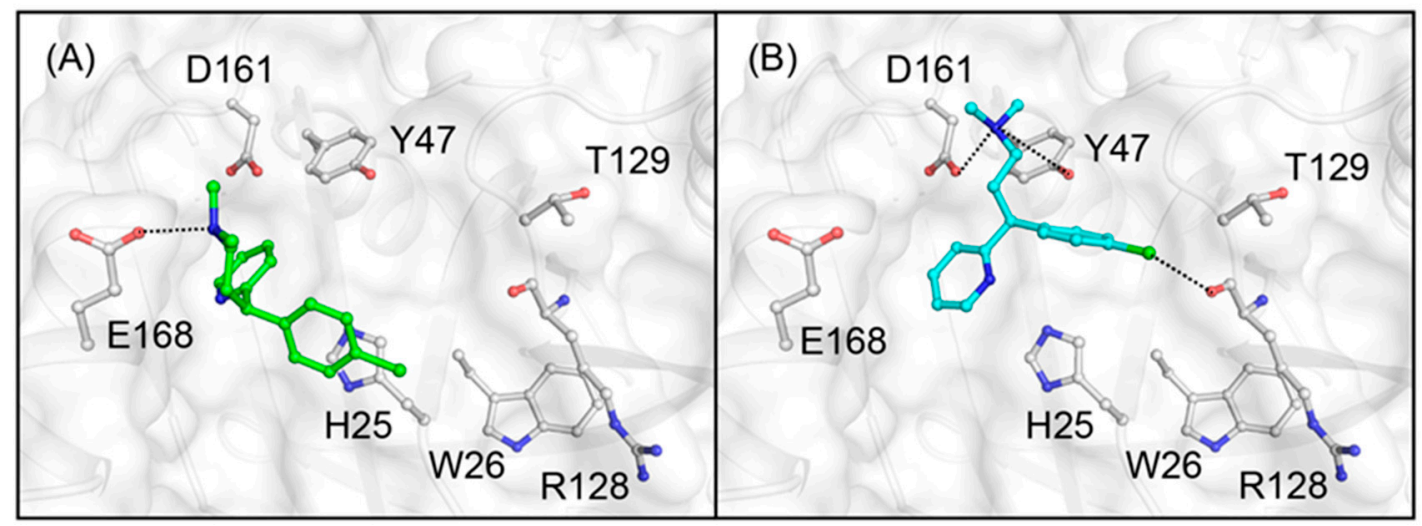

Figure 4. Simulated docking structures of cAGP with (A) (R)-chlorpheniramine and (B) (S)-chlorpheniramine. The docked compounds were represented as in Figure 3. Besides, the same colors were used for nitrogen and oxygen atoms, and the remarked amino acid residues as in Figure 3. Feasible interactions were depicted as dotted lines.

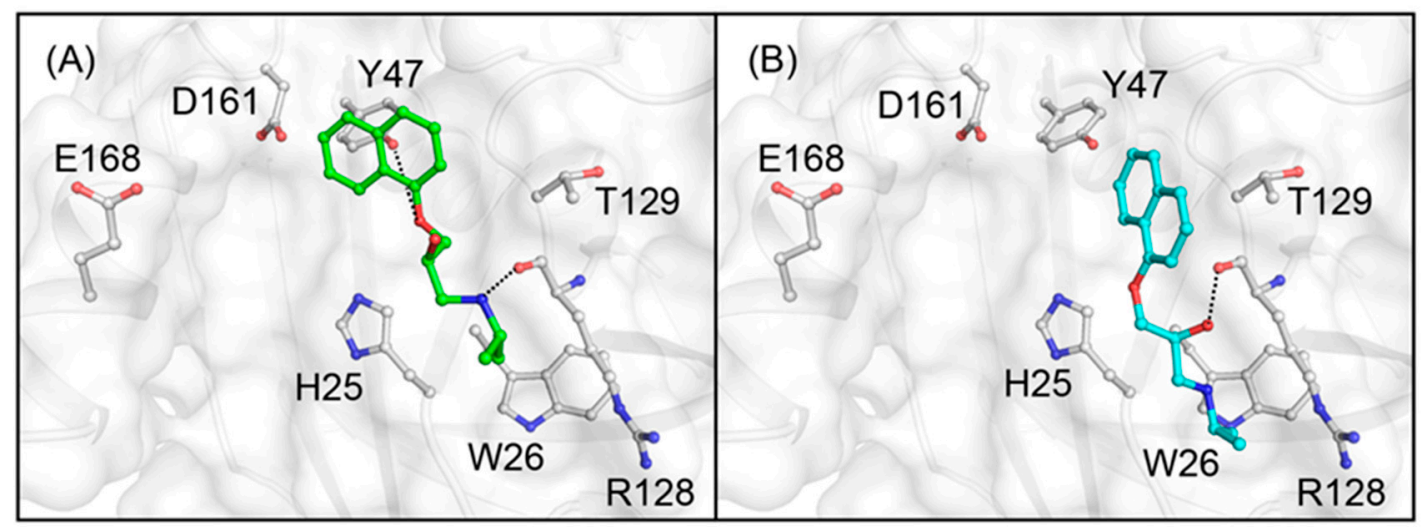

Figure 5. Simulated docking structures of cAGP with (A) (R)-propranolol and (B) (S)-propranolol. The docked compounds were represented as in Figure 3. Besides, the same colors were used for nitrogen and oxygen atoms, and the remarked amino acid residues as in Figure 3. Feasible interactions were depicted as dotted lines.

\section{Discussion}

Figure 6 shows sequence alignments of F1*S and A variant hAGPs and cAGP along with the highlighted amino acids, which consist of feasible ligand-binding sites. The F1*S variant hAGP has lobs I, II and III as the ligand-binding sites, while the A variant hAGP has only lobs I and II. Figure 7 shows crystal structures of F1*S (left; pdbid, 3kq0) and A (center; pdbid, 3apu) variant hAGPs and a built model structure of cAGP (right). These indicate that the ligand-binding sites of hAGP and cAGP are totally different: A lot of ligands bound almost the same sites on hAGP, lobes I-III, while the three ligands, benzoin, chlorpheniramine and propranolol, bound the cavity near a W26 region on cAGP. The hydrophobic cavities lined with H25, W26, Y47, R128, T129, D161 and E168, which contribute electrostatic or hydrogen bonding interactions, were responsible for chiral resolution of the studied compounds on cAGP. 


\begin{tabular}{|c|c|c|c|c|}
\hline $\mathrm{F} 1{ }^{\star} \mathrm{S}$ hAGP & - QIPLCANLV & PVPITNATLD & QITGKWFYIA & SAFRNEEYNK SVQEIQATFF \\
\hline A $h A G P$ & MQIPLCANLV & PVPITNATLD & RITGKWFYIA & SAFRNEEYNK SVQEIQATFF \\
\hline CAGP & TESPTCAPLV & TADMDNATVD & RLLGHWVYIM & GASQYPPHMA EMRELKYATF \\
\hline $\mathrm{F} 1 * \mathrm{~S}$ hAGP & YFTPNKTEDT & IFLREYQTRQ & DQCIYN-TTY & LNVQRENGTI SRYVGGQEHF \\
\hline A hAGP & YFTPNKTEDT & IFLREYQTRQ & $\mathrm{NQCFYN}-\mathrm{SSY}$ & LNVQRENGTV SRYEGGREHV \\
\hline CAGP & TLFPGSHEDE & FNVTEIMRLN & ETCVVKNSSK & IHVFRHNSTL THEDG---QV \\
\hline $\mathrm{F} 1 * \mathrm{~S}$ hAGP & AHLLI LRDTK & TYMLAFDVND & E KNWGLSVYA & DKPETTKEQL GEFYEALDCL \\
\hline A $h A G P$ & AHLLFLRDTK & TLMFGSYLDD & EKNWGLS FYA & DKPETTKEQL GEFYEALDCL \\
\hline Chicken & VSMAELIHSD & KDLF I LKH FK & DNHVGLSLSA & RTAEVTKEQL EEFEAQLRCH \\
\hline $\mathrm{F} 1{ }^{*} \mathrm{~S}$ hAGP & RIPKS DVVYT & DWKKDKCEPL & EKQHEKERKQ & EEGESAWSHPQFEK \\
\hline A $h A G P$ & RIPRS DVMYT & DWKKDKCEPL & EKQHEKERKQ & E EGESHHHHH H \\
\hline CAGP & GFKLEEAFIT & $S P K D A C P A A G$ & EETGEGSAAP & $A E P Q L G$ \\
\hline
\end{tabular}

Figure 6. Sequence alignments of $\mathrm{F} 1{ }^{*} \mathrm{~S}$ and A variant hAGPs and cAGP. The cavities in hAGP were called as lobes being highlighted in green for lobe I, orange for lobe II and cyan for lobe III. The amino acids, which consisted of feasible ligand-binding sites in this work, were colored in magenta.
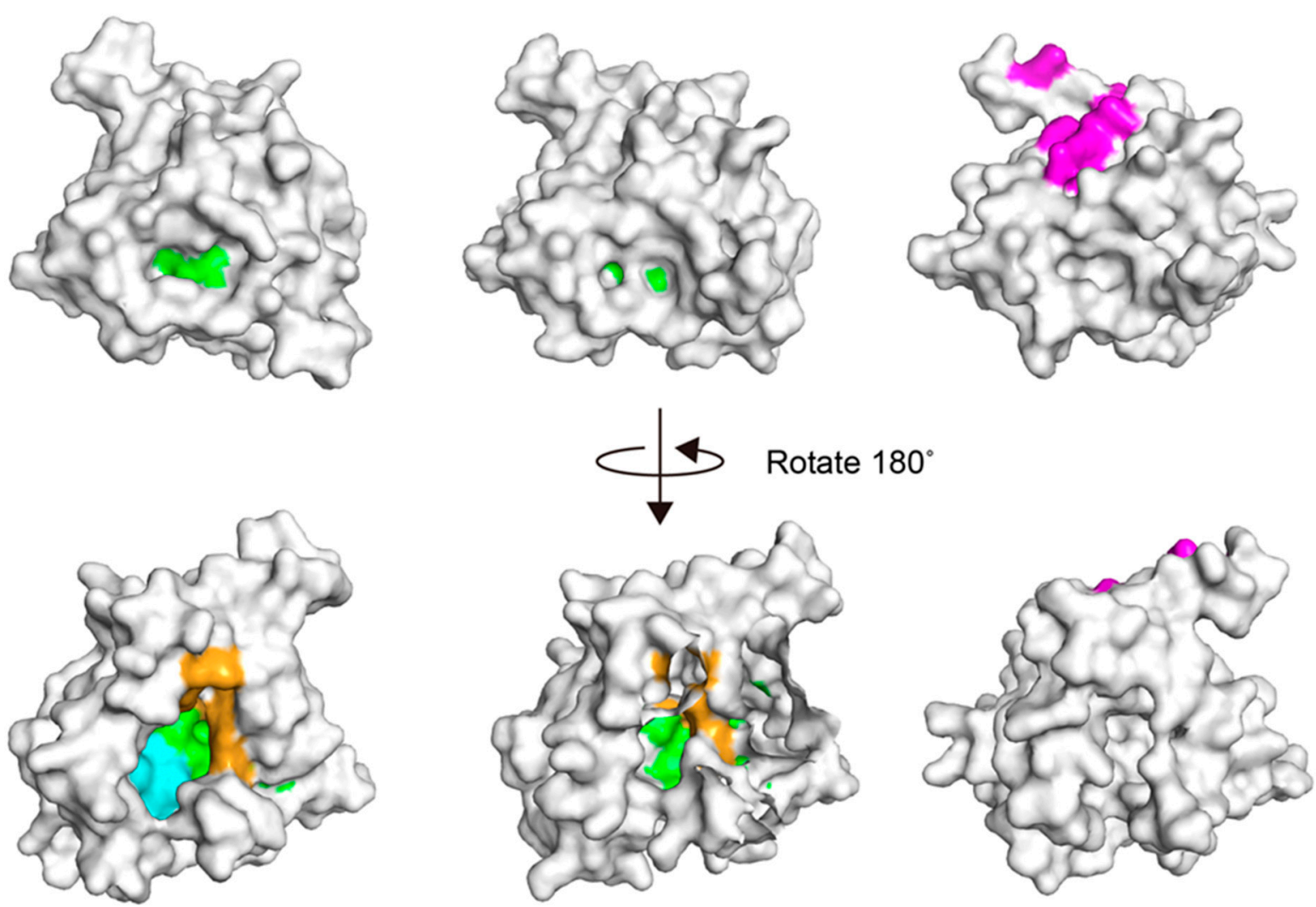

Figure 7. Crystal structures of F1*S (left) and A (middle) variant hAGPs and a built model structure of cAGP (right). The A variant of cAGP was only showed one of subunits. The highlighted residues in the Figure 6 were colored in the structures. 
In docking simulations of studied enantiomers into the generated model structure of cAGP, the $(R)$-benzoin, $(S)$-chlorpheniramine or $(R)$-propranolol binding was more favorable than its enantiomer. These results correlate well with the chromatographic results that $(R)$-benzoin, $(S)$-chlorpheniramine or $(R)$-propranolol is more retained than its enantiomer on a cAGP column. Furthermore, chiral resolution of propranolol was completely lost on a W26-modified cAGP column. This could be due to the small changes in the position of the side chains of amino acid residues in chiral binding sites near W26. However, on a W26-modified cAGP column chiral resolution of benzoin and chlorpheniramine was still attained. Because their chiral binding sites were a little away from W26. Previously, we considered that there existed at least two chiral binding sites on cAGP. Taking into account the results of docking simulations, we conclude that the chiral binding sites for the studied compounds are near a W26 region.

In docking simulations of chlorpheniramine enantiomers, electrostatic interactions between amino groups of $(R)$ - and (S)-chlorpheniramine with carboxyl groups of E168 and D161 of cAGP, respectively, worked for chiral recognition. It was reported that the retention and enantioseparation factors increased with an increase in mobile-phase $\mathrm{pH}$ on a crude ovomucoid (contaminated with cAGP) column [36]. This suggests that the ionic interactions work for the retention and chiral recognition of chlorpheniramine enantiomers. On the other hand, in docking simulations of propranolol enantiomers, plausible ionicinteractions between an amino group of each propranolol enantiomer with a carboxyl group of cAGP were not found. Consequently, the results of docking simulations well coincide with the data that the retention factor increases with an increase in mobile-phase $\mathrm{pH}$ on a crude ovomucoid (contaminated with cAGP) column, but that the enantioseparation factor remains unchanged [49]. The retention of propranolol was governed by nonspecific interactions of propranolol with cAGP, while the enantioselectivity came from the specific interactions of propranolol in the chiral binding site near W26. We also constructed the model structure of W26-modified cAGP with 2-ntirophenylsulfenyl group (data not shown). Although the generated model structure was less reliable, it seemed that the introduced 2-ntirophenylsulfenyl group could yield enhanced rigidity for the cAGP structure, and that small changes in the position of the side chains of amino acid residues occurred. These changes might result in the loss of enantioselectivity of propranolol on the W26modified column.

\section{Conclusions}

Based on chromatographic properties of native and W26-modified cAGP columns and docking simulations of studied compounds into the generated model structure of cAGP, the chiral binding sites were located on cAGP for the first time and the chiral binding mechanism was discussed. On cAGP, there existed a hydrophobic cavity lined with H25, W26, Y47, R128, T129, D161 and E168. Benzoin and chlorpheniramine enantiomers interacted with cAGP at almost the same sites a little away from W26, while propranolol enantiomers docked in the relatively small cliff, slightly shifting toward H25 and W26. Furthermore, in addition to hydrophobic interactions, ionic interactions between amino groups of chlorpheniramine enantiomers and a carboxy group of D161 or E168 played an important role in the chiral recognition, while hydrophobic interactions and hydrogen bonding interactions worked for the chiral recognition of benzoin and propranolol enantiomers.

Author Contributions: Conceptualization, J.H. and T.Y.; methodology, J.H, T.Y., H.T. and M.A.; formal analysis, J.H, T.Y., H.T. and M.A.; investigation, J.H, T.Y., H.T. and M.A.; resources, J.H.; data curation, J.H, T.Y., H.T. and M.A.; writing—original draft preparation, J.H.; writing-review and editing, J.H, T.Y., H.T. and M.A.; visualization, T.Y., H.T. and M.A.; supervision, J.H.; project administration, J.H. and T.Y.; funding acquisition, J.H. All authors have read and agreed to the published version of the manuscript.

Funding: This work was partially supported by a funding from Daicel (Tokyo, Japan).

Data Availability Statement: Not applicable. 
Acknowledgments: Not applicable.

Conflicts of Interest: The authors declare no conflict of interest.

\section{References}

1. Haginaka, J. Mechanistic Aspects of Chiral Recognition on Protein-Based Stationary Phases. Adv. Chromatogr. 2011, 49, 37-69. [CrossRef]

2. Bi, C.; Zheng, X.; Azaria, S.; Beeram, S.; Li, Z.; Hage, D.S. Chromatographic Studies of Protein-Based Chiral Separations. Separations 2016, 3, 27. [CrossRef] [PubMed]

3. Allenmark, S.; Bomgren, B.; Borén, H. Direct Liquid Chromatographic Separation of Enantiomers on Immobilized Protein Stationary Phases. IV. Molecular Interaction Forces and Retention Behaviour in Chromatography on Bovine Serum Albumin as a Stationary Phase. J. Chromatogr. 1984, 316, 617-624. [CrossRef]

4. Domenici, E.; Bertucci, C.; Salvadori, P.; Felix, G.; Cahagne, I.; Motellier, S.; Wainer, I.W. Synthesis and Chromatographic Properties of an HPLC Chiral Stationary Phase Based upon Human Serum Albumin. Chromatographia 1990, 29, 170-176. [CrossRef]

5. Hermansson, J. Direct Liquid Chromatographic Resolution of Racemic Drugs Using $\alpha 1$-Acid Glycoprotein as the Chiral Stationary Phase. J. Chromatogr. A 1983, 269, 71-80. [CrossRef]

6. Haginaka, J.; Seyama, C.; Kanasugi, N. The Absence of Chiral Recognition Ability in Ovomucoid: Ovoglycoprotein-bonded HPLC Stationary Phases for Chiral Recognition. Anal. Chem. 1995, 67, 2539-2547. [CrossRef]

7. Sadakane, Y.; Matsunaga, H.; Nakagomi, K.; Hatanaka, Y.; Haginaka, J. Protein Domain of Chicken $\alpha 1$-Acid Glycoprotein is Responsible for Chiral Recognition Ability. Biochem. Biophys. Res. Commun. 2002, 295, 587-590. [CrossRef]

8. Thelohan, S.; Jadaud, P.; Wainer, I.W. Immobilized Enzymes as Chromatographic Phases for HPLC: The Chromatography of Free and Derivatized Amino Acids on Immobilized Trypsin. Chromatographia 1989, 28, 551-555. [CrossRef]

9. Erlandsson, P.; Marle, I.; Hansson, L.; Isaksson, R.; Petterson, C.; Petterson, G. Immobilized Cellulase (CBH I) as a Chiral Stationary Phase for Direct Resolution of Enantiomers. J. Am. Chem. Soc. 1990, 112, 4573-4574. [CrossRef]

10. Haginaka, J.; Miyano, Y.; Saizen, Y.; Seyama, C.; Murashima, T. Separation of Enantiomers on a Pepsin-bonded Column. J. Chromatogr. A 1995, 708, 161-168. [CrossRef]

11. Nystrom, A.; Strandberg, A.; Aspergren, A.; Behr, S.; Karlsson, A. Use of Immobilized Amyloglucosidase as Chiral Selector in Chromatography. Immobilization and Performance in Liquid Chromatography. Chromatographia 1999, 50, 209-214. [CrossRef]

12. Hofstetter, H.; Hofstetter, O. Antibodies as Tailor-made Chiral Selectors for Detection and Separation of Stereoisomers. Trends Anal. Chem. 2005, 24, 869-879. [CrossRef]

13. Massolini, G.; de Lorenzi, E.; Calleri, E.; Bertucci, C.; Monaco, H.L.; Perduca, M.; Caccialanza, G.; Wainer, I.W. Properties of a Stationary Phase Based on Immobilized Chicken Liver Basic Fatty Acid-binding Protein. J. Chromatogr. B 2001, 751, 117-130. [CrossRef]

14. Schmid, K.; Nimerg, R.B.; Kimura, A.; Yamaguchi, H.; Binette, J.P. The Carbohydrate Units of Human Plasma Alpha1-acid Glycoprotein. Biochim. Biophys. Acta 1977, 492, 291-302. [CrossRef]

15. Luo, Z.; Lei, H.; Sun, Y.; Liu, X.; Su, D.F. Orosomucoid, an Acute Response Protein with Multiple Modulating Activities. J. Physiol. Biochem. 2015, 71, 329-340. [CrossRef]

16. Kremer, J.M.; Wilting, J.; Janssen, L.H. Drug Binding to Human Alpha-1-acid Glycoprotein in Health and Disease. Pharmacol. Rev. 1988, 40, 1-47.

17. Israili, Z.H.; Dayton, P.G. Human Alpha-1-Glycoprotein and Its Interactions with Drugs. Drug Metab. Rev. 2001, 33, 161-235. [CrossRef]

18. Dente, L.; Pizza, M.G.; Metspalu, A.; Cortese, R. Structure and Expression of the Genes Coding for Human Alpha 1-acid Glycoprotein. EMBO J. 1987, 6, 2289-2296. [CrossRef] [PubMed]

19. Eap, C.B.; Baumann, P. The Genetic Polymorphism of Human Alpha 1-acid Glycoprotein. Prog. Clin. Biol. Res. 1989, 300, 111-125.

20. Yuasa, I.; Weidinger, S.; Umetsu, K.; Suenaga, K.; Ishimoto, G.; Eap, B.C.; Duche, J.C.; Baumann, P. Orosomucoid System: 17 Additional Orosomucoid Variants and Proposal for a New Nomenclature. Vox Sang. 1993, 64, 47-55. [CrossRef]

21. Schönfeld, D.L.; Ravelli, R.B.; Mueller, U.; Skerra, A. The 1.8-Å Crystal Structure of Alpha1-acid Glycoprotein (Orosomucoid) Solved by UV RIP Reveals the Broad Drug-binding Activity of This Human Plasma Lipocalin. J. Mol. Biol. 2008, 384, 393-405. [CrossRef]

22. Nishi, K.; Ono, T.; Nakamura, T.; Fukunaga, N.; Izumi, M.; Watanabe, H.; Suenaga, A.; Maruyama, T.; Yamagata, Y.; Curry, S.; et al. Structural Insights into Differences in Drug-binding Selectivity between Two Forms of Human Alpha1-acid Glycoprotein Genetic Variants, the A and F1*S Forms. J. Biol. Chem. 2011, 286, 14427-14434. [CrossRef]

23. Zsila, F.; Bikádi, Z.; Simonyi, M. Induced Circular Dichroism Spectra Reveal Binding of the Antiinflammatory Curcumin to Human Alpha1-acid Glycoprotein. Bioorg. Med. Chem. 2004, 12, 3239-3245. [CrossRef]

24. Hazai, E.; Visy, J.; Fitos, I.; Bikádi, Z.; Simonyi, M. Selective Binding of Coumarin Enantiomers to Human Apha1-acid Glycoprotein Genetic Variants. Bioorg. Med. Chem. 2006, 14, 1959-1965. [CrossRef] [PubMed]

25. Azad, M.A.; Huang, J.X.; Cooper, M.A.; Roberts, K.D.; Thompson, P.E.; Nation, R.L.; Li, J.; Velkov, T. Structure-activity Relationships for the Binding of Polymyxins with Human $\alpha$-1-Acid Glycoprotein. Biochem Pharmacol. 2012, 84, $278-291$. [CrossRef] 
26. Huang, J.X.; Cooper, M.A.; Baker, M.A.; Azad, M.A.; Nation, R.L.; Li, J.; Velkov, T. Drug-binding Energetics of Human $\alpha-1-A c i d$ Glycoprotein Assessed by Isothermal Titration Calorimetry and Molecular Docking Simulations. J. Mol. Recognit. 2012, 25, 642-656. [CrossRef] [PubMed]

27. Ajmal, M.R.; Abdelhameed, A.S.; Alam, P.; Khan, R.H. Interaction of New Kinase Inhibitors Cabozantinib and Tofacitinib with Human Serum Alpha-1 Acid Glycoprotein. A Comprehensive Spectroscopic and Molecular Docking Approach. Spectrochim. Acta A Mol. Biomol. Spectrosc. 2016, 159, 199-208. [CrossRef]

28. Ajmal, M.R.; Almutairi, F.; Zaidi, N.; Alam, P.; Siddiqi, M.K.; Khan, M.V.; Zaman, M.; Ishtikhar, M.; Khan, R.H. Biophysical Insights into the Interaction of Clofazimine with Human Alpha 1-acid Glycoprotein: A Multitechnique Approach. J. Biomol. Struct. Dyn. 2019, 37, 1390-1401. [CrossRef] [PubMed]

29. Nishi, K.; Sakurama, K.; Kobashigawa, Y.; Morioka, H.; Udo, N.; Hashimoto, M.; Imoto, S.; Yamasaki, K.; Otagiri, M. Interaction of Aripiprazole With Human $\alpha_{1}$-Acid Glycoprotein. J. Pharm. Sci. 2019, 108, 3911-3916. [CrossRef]

30. Wang, B.L.; Kou, S.B.; Lin, Z.Y.; Shi, J.H.; Liu, Y.X. Insights on the Interaction Mechanism of Brigatinib to Human $\alpha-1-A c i d$ Glycoprotein: Experimental and Computational Approaches. Int. J. Biolog. Macromolec. 2020, 157, 340-349. [CrossRef] [PubMed]

31. Miyoshi, T.; Sukimoto, K.; Otagiri, M. Investigation of the Interaction Mode of Phenothiazine Neuroleptics with Apha 1-acid Glycoprotein. J. Pharm. Pharmacol. 1992, 44, 28-33. [CrossRef] [PubMed]

32. Zsila, F.; Iwao, Y. The Drug Binding Site of Human Alpha1-acid Glycoprotein: Insight from Induced Circular Dichroism and Electronic Absorption Spectra. Biochim. Biophys. Acta. 2007, 1770, 797-809. [CrossRef] [PubMed]

33. Katsuki, M.; Chuang, V.T.; Nishi, K.; Kawahara, K.; Nakayama, H.; Yamaotsu, N.; Hirono, S.; Otagiri, M. Use of Photoaffinity Labeling and Site-directed Mutagenesis for Identification of the Key residue Responsible for Extraordinarily High Affinity Binding of UCN-01 in Human Alpha1-acid Glycoprotein. J. Biol. Chem. 2005, 280, 1384-1391. [CrossRef] [PubMed]

34. Becker, B.A.; Larive, C.K. Probing the Binding of Propranolol Enantiomers to $\alpha 1$-Acid Glycoprotein with Ligand-Detected NMR Experiments. J. Phys. Chem. B 2008, 112, 13581-13587. [CrossRef]

35. Miwa, T.; Ichikawa, M.; Tsuno, M.; Hattori, T.; Miyakawa, T.; Kayano, M.; Miyake, Y. Direct Liquid Chromatographic Resolution of Racemic Compounds. Use of Ovomucoid as a Column Ligand. Chem. Pharm. Bull. 1987, 35, 682-686. [CrossRef]

36. Miwa, T.; Miyakawa, T.; Kayano, M.; Miyake, Y. Application of an Ovomucoid-conjugated Column for the Optical Resolution of Some Pharmaceutically Important Compounds. J. Chromatogr. A 1987, 408, 316-322. [CrossRef]

37. Matsunaga, H.; Sadakane, Y.; Haginaka, J. Identification of Disulfide Bonds and Site-specific Glycosylation in Chicken Alpha1-acid Glycoprotein by Matrix-assisted Laser Desorption Ionization Time-of-flight Mass Spectrometry. Anal. Biochem. 2004, 331, 358-363. [CrossRef] [PubMed]

38. Matsunaga, H.; Haginaka, J. Investigation of Chiral Recognition Mechanism on Chicken Alpha1-acid Glycoprotein Using Separation System. J. Chromatogr. A 2006, 1106, 124-130. [CrossRef]

39. Zsila, F.; Matsunaga, H.; Bikádi, Z.; Haginaka, J. Multiple Ligand-binding Properties of the Lipocalin Member Chicken Alpha1acid Glycoprotein Studied by Circular Dichroism and Electronic Absorption Spectroscopy: The Essential Role of the Conserved Tryptophan Residue. Biochim. Biophys. Acta 2006, 1760, 1248-1273. [CrossRef] [PubMed]

40. Chattopadhyay, A.; Tian, T.; Kortum, L.; Hage, D.S. Development of Tryptophan-modified Human Serum Albumin Columns for Site-specific Studies of Drug-protein Interactions by High-performance Affinity Chromatography. J. Chromatogr. B 1998, 715, 183-190. [CrossRef]

41. Haginaka, J.; Takehira, H. Separation of Enantiomers on a Chiral Stationary Phase Based on Ovoglycoprotein. I. Influences of the Pore Size of Base Silica Materials and Bound Protein Amounts on Chiral Resolution. J. Chromatogr. A 1977, 773, 85-91. [CrossRef]

42. Waterhouse, A.; Bertoni, M.; Bienert, S.; Studer, G.; Tauriello, G.; Gumienny, R.; Heer, F.T.; de Beer, T.; Rempfer, C.; Bordoli, L.; et al. SWISS-MODEL: Homology Modelling of Protein Structures and Complexes. Nucleic Acids Res. 2018, 46, W296-W303. [CrossRef] [PubMed]

43. Studer, G.; Tauriello, G.; Bienert, S.; Biasini, M.; Johner, N.; Schwede, T. ProMod3-A Versatile Homology Modelling Toolbox. PLoS Comput. Biol. 2021, 17, e1008667. [CrossRef]

44. Yang, J.; Zhang, Y. I-TASSER Server: New Development for Protein Structure and Function Predictions. Nucleic Acids Res. 2015, 43, W174-W181. [CrossRef] [PubMed]

45. Bharatham, N.; Bharatham, K.; Shelat, A.A.; Bashford, D. Ligand Binding Mode Prediction by Docking: Mdm2/Mdmx Inhibitors as a Case Study. J. Chem. Inf. Model. 2014, 54, 648-659. [CrossRef]

46. Kumar, G.K.; Prasanna, G.; Marimuthu, T.; Saraswathi, N.T. Structural Basis for Complementary and Alternative Medicine: Phytochemical Interaction with Non-structural Protein 2 Protease-a Reverse Engineering Strategy. Chin. J. Integr. Med. 2015, 21, 445-452. [CrossRef] [PubMed]

47. Ramakrishnan, P.; Pavan Kumar, T.; Saraswathy, G.R.; Sujatha, S. In Silico Evaluation of Drugs Used in Treatment of Oral Lichen Planus. J. Oral Pathol. Med. 2020, 49, 926-932. [CrossRef]

48. Friesner, R.A.; Banks, J.L.; Murphy, R.B.; Halgren, T.A.; Klicic, J.J.; Mainz, D.T.; Repasky, M.P.; Knoll, E.H.; Shelley, M.; Perry, J.K.; et al. Glide: A New Approach for Rapid, Accurate Docking and Scoring. 1. Method and Assessment of Docking Accuracy. J. Med. Chem. 2004, 47, 1739-1749. [CrossRef] [PubMed]

49. Haginaka, J.; Wakai, J.; Takahashi, K.; Yasuda, H.; Katagi, T. Chiral Separation of Propranolol and Its Ester Derivatives on an Ovomucoid-bonded Silica: Influence of $\mathrm{pH}$, Ionic Strength and Organic Modifier on Retention, Enantioselectivity and Enantiomeric Elution Order. Chromatographia 1990, 29, 587-592. [CrossRef] 